\title{
Rational thermostabilisation of four-helix bundle dimeric de novo proteins
}

\author{
Shin Irumagawa ${ }^{1,2,3}$, Kaito Kobayashi ${ }^{4}$, Yutaka Saito ${ }^{4,5,6}$, Takeshi Miyata ${ }^{7}$, Mitsuo Umetsu ${ }^{8}$, \\ Tomoshi Kameda ${ }^{4}$ \& Ryoichi Arai ${ }^{1,2,3 凶}$
}

The stability of proteins is an important factor for industrial and medical applications. Improving protein stability is one of the main subjects in protein engineering. In a previous study, we improved the stability of a four-helix bundle dimeric de novo protein (WA20) by five mutations. The stabilised mutant (H26L/G28S/N34L/V71L/E78L, SUWA) showed an extremely high denaturation midpoint temperature $\left(T_{\mathrm{m}}\right)$. Although SUWA is a remarkably hyperstable protein, in protein design and engineering, it is an attractive challenge to rationally explore more stable mutants. In this study, we predicted stabilising mutations of WA20 by in silico saturation mutagenesis and molecular dynamics simulation, and experimentally confirmed three stabilising mutations of WA20 (N22A, N22E, and H86K). The stability of a double mutant (N22A/H86K, rationally optimised WA20, ROWA) was greatly improved compared with WA20 $\left(\Delta T_{\mathrm{m}}=10.6^{\circ} \mathrm{C}\right)$. The model structures suggested that N22A enhances the stability of the $\alpha$-helices and N22E and H86K contribute to salt-bridge formation for protein stabilisation. These mutations were also added to SUWA and improved its $T_{\mathrm{m}}$. Remarkably, the most stable mutant of SUWA (N22E/H86K, rationally optimised SUWA, ROSA) showed the highest $T_{\mathrm{m}}$ $\left(129.0^{\circ} \mathrm{C}\right)$. These new thermostable mutants will be useful as a component of protein nanobuilding blocks to construct supramolecular protein complexes.

Improving the stability of proteins is one of main subjects in protein engineering. Development of methods to stabilise proteins will contribute to medical and industrial applications. For many years, stabilisation of various proteins has been studied in the fields of protein engineering ${ }^{1-6}$. Many studies on protein stabilisation by mutagenesis have been reported, but investigating sufficient mutations of amino-acid residues requires enormous effort and cost for experiments. Several computational methods have been used to rationally predict stabilising mutations ${ }^{7,8}$. Because proteins are dynamic molecules in solution and molecular dynamics (MD) simulation provides information about the dynamic behaviour of molecules in variable environments in silico, MD simulation is useful for designing stabilised proteins ${ }^{9}$.

Protein design is also a main and hot topic in protein engineering ${ }^{10-13}$. As a semirational approach for protein design, the binary code strategy was developed to construct libraries of novel polypeptides (de novo proteins) that would fold into predetermined structures ${ }^{14}$. Using secondary structure motifs with binary patterns of polar and nonpolar residues, de novo proteins with $\alpha$-helices or $\beta$-sheets have been successfully created without reference to natural protein sequences. WA20 is one of the de novo proteins obtained from a library of binary patterned four-helix bundles ${ }^{15}$. Previously the crystal structure of WA20 was solved, revealing an intermolecularly folded dimeric four-helix bundle (PDB ID: $3 \mathrm{VJF})^{16}$ with a bisecting $\mathrm{U}$ topology ${ }^{17}$. Moreover, utilising the characteristic dimeric structure of WA20 as a component, protein nanobuilding blocks (PN-Blocks) ${ }^{18,19}$ were developed to construct self-assembled supramolecular nanostructures: WA20-foldon, constructed by fusing dimeric WA20 to a trimeric foldon domain of T4 phage fibritin formed several types of self-assembled nanoarchitectures, including

\footnotetext{
${ }^{1}$ Department of Science and Technology, Graduate School of Medicine, Science and Technology, Shinshu University, Ueda, Nagano 386-8567, Japan. ${ }^{2}$ Department of Biomolecular Innovation, Institute for Biomedical Sciences, Interdisciplinary Cluster for Cutting Edge Research, Shinshu University, Matsumoto, Nagano 390-8621, Japan. ${ }^{3}$ Department of Applied Biology, Faculty of Textile Science and Technology, Shinshu University, Ueda, Nagano 386-8567, Japan. ${ }^{4}$ Artificial Intelligence Research Center, National Institute of Advanced Industrial Science and Technology (AIST), Tokyo 135-0064, Japan. ${ }^{5}$ AIST-Waseda University Computational Bio Big-Data Open Innovation Laboratory (CBBD-OIL), Tokyo 169-8555, Japan. ${ }^{6}$ Graduate School of Frontier Sciences, The University of Tokyo, Kashiwa, Chiba 277-8561, Japan. ${ }^{7}$ Department of Biochemistry and Biotechnology, Faculty of Agriculture, Kagoshima University, Kagoshima 890-0065, Japan. ${ }^{8}$ Department of Biomolecular Engineering, Graduate School of Engineering, Tohoku University, Sendai 980-8579, Japan. ${ }^{\circledR}$ email: rarai@shinshu-u.ac.jp
} 


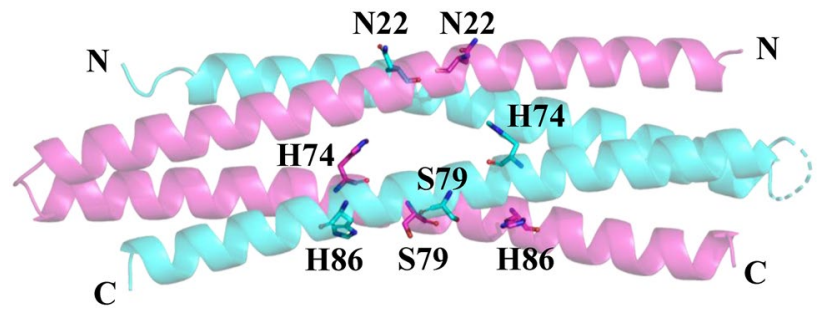

Figure 1. Target residues for mutations to stabilise the de novo protein WA20 in the present study. The target amino acid residues are shown as sticks. Chains A and B of the crystal structure of WA20 (PDB: $3 \mathrm{VJF})^{16}$ are shown in magenta and cyan, respectively. The images were created using open- source PyMOL, version 2.4 (https://github.com/schrodinger/pymol-open-source).

a barrel-like hexamer and a tetrahedrally shaped dodecamer ${ }^{18}$. In addition, extender protein nanobuilding blocks (ePN-Blocks) was constructed by tandemly joining two copies of WA20 with various linkers ${ }^{19}$. The ePN-Blocks self-assembled into cyclised and extended chain-type nanostructures.

With the long-term goal of producing nanostructures with extremely high stabilities for applications in nanotechnology, we were motivated to stabilise the designed protein WA20, a main component of PN-Blocks. Recently, we succeeded in dramatically improving the stability of WA20 by introducing five amino acid substitutions (H26L, G28S, N34L, V71L, and E78L) ${ }^{20}$ to enhance the hydrophobic core and a-helix stability based on the WA20 structure. This mutant, which is called super WA20 (SUWA), showed an extremely high denaturation midpoint temperature $\left(T_{\mathrm{m}}\right)$ above the boiling point of water.

Although SUWA is a remarkably hyperstable protein, in protein engineering, it is an attractive challenge to rationally explore more stable mutants than the de novo designed proteins WA20 and SUWA. In this study, we performed rational design of new mutations to stabilise WA20 and SUWA by in silico mutagenesis and MD simulation. Three mutations (N22A, N22E, and H86K) were found to improve $T_{\mathrm{m}}$ of WA20. A double mutant of WA20 (N22A/H86K, which is called rationally optimised WA20, ROWA) greatly improved $T_{\mathrm{m}}$ by $10.6{ }^{\circ} \mathrm{C}$. Moreover, these mutations improved the thermostability of SUWA. In particular, a double mutant of SUWA (N22E/H86K, which is called rationally optimised SUWA, ROSA) showed the highest $T_{\mathrm{m}}$ of $129.0^{\circ} \mathrm{C}$. In addition, the ROWA and ROSA oligomers were characterised by size exclusion chromatography-multi-angle light scattering (SEC-MALS) and small-angle X-ray scattering (SAXS).

\section{Results}

Prediction of mutations to stabilise WA20. For soluble and stable proteins, generally, most of the amino acid residues on the surface of the proteins are hydrophilic and most of the residues inside the proteins are hydrophobic. However, in some cases, there are some unusual residues, which are hydrophilic residues buried inside the protein, that can be potentially optimised. Therefore, to select target residues for mutations, we searched for hydrophilic residues buried in the WA20 protein structure based on the accessible surface area (ASA) per residue (Supplementary Table S1). Five hydrophilic residues buried inside the structure of WA20 (H26, H74, E78, S79, and H86) were found based on small ASA ratios (ASA ratio $\leq 0.11$ in both the A and B chains). In addition, we chose two target residues (N22 and N34) on the interface of the a-helices to potentially enhance the helix-helix interactions (Supplementary Fig. S1). In a previous study of SUWA ${ }^{20}$, we tested some mutations (H26L/E78L and N34L) at these target residue sites. The double mutation H26L/E78L significantly improved $T_{\mathrm{m}}$ of WA20 by $26^{\circ} \mathrm{C}$ and the single mutation $\mathrm{N} 34 \mathrm{~L}$ improved $T_{\mathrm{m}}$ by $10^{\circ} \mathrm{C}^{20}$. Therefore, in the present study, we focused on the target residues (N22, H74, S79, and H86, Fig. 1) that we did not test previously.

Saturation mutagenesis is an exhaustive search for all possible mutations of 19 amino acids at a target site, but enormous effort and cost are required for experiments. Hence, we attempted to predict stabilising mutations using MD simulation. First, we performed high-temperature MD simulations of WA20 and the mutants constructed in a previous study (G28S, N34L, V71L, H26L/E78L, H26L/G28S/V71L/E78L, and SUWA) ${ }^{20}$. The root mean square deviations (RMSDs) between the initial structures and the structures after high-temperature MD simulations were calculated to evaluate the degree of protein unfolding. The RMSD values of WA20 and the mutants showed a strong negative correlation with $T_{\mathrm{m}}$ of the mutants $\left(r=-0.966, p=5.461 \times 10^{-5}\right.$, Supplementary Fig. S2), suggesting that RMSD is a useful indicator to evaluate the stability of mutants. Therefore, we performed high-temperature MD simulations of all of the possible single mutants and the original WA20 (i.e., in silico saturation mutagenesis) at the four target residue sites (N22, H74, S79, and H86). Based on the MD simulation results, we selected eight mutations of WA20 with small RMSD values (N22A, N22E, N22K, N22L, H86K, H86S, H74V, and S79F, Supplementary Fig. S3) for subsequent experiments. At the N22 site, the N22G mutation showed the smallest RMSD value, but we did not adopt N22G because the RMSD values of N22G showed relatively large variability and glycine is generally unfavourable in an $\alpha$-helix of proteins ${ }^{21}$. Because the H74 and S79 sites are close, we constructed the double mutant of H74V/S79F with one oligo-DNA primer for the site-directed mutations.

Development of stabilised mutants of WA20. WA20 and the mutant proteins were expressed in Escherichia coli and purified by immobilised metal ion affinity chromatography (IMAC) (Supplementary 
A

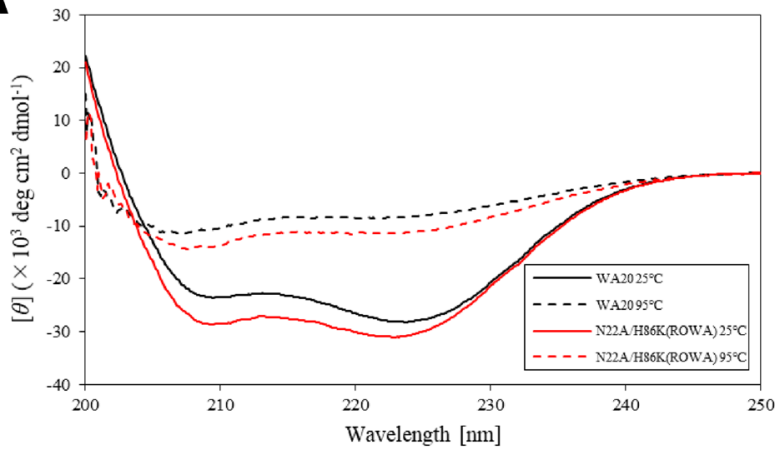

C

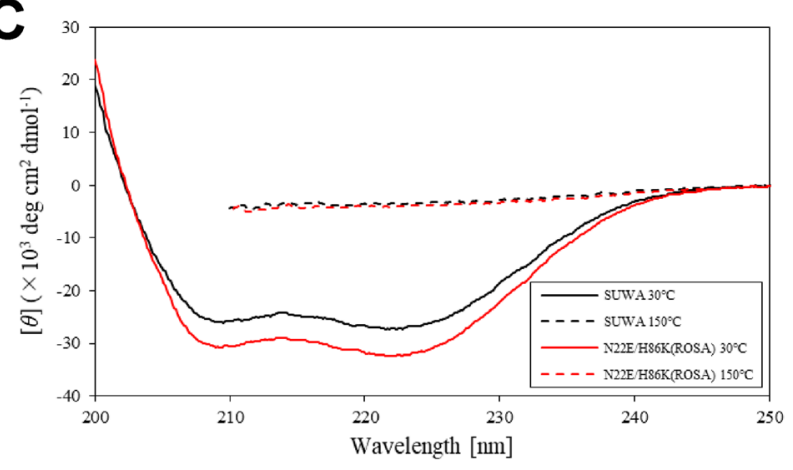

B

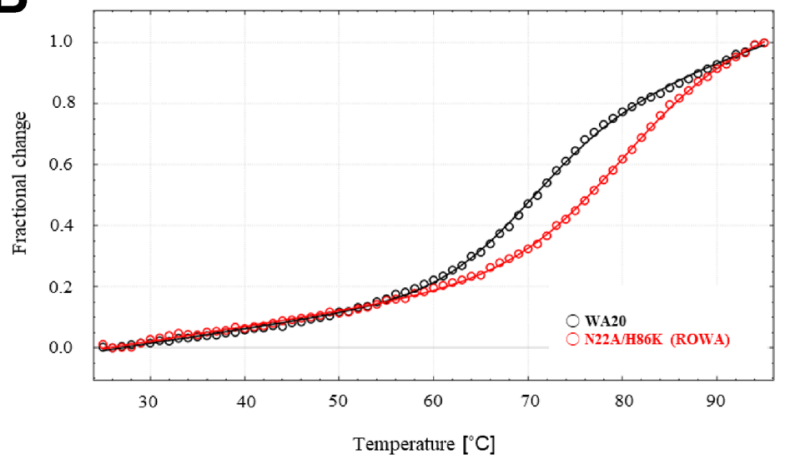

D

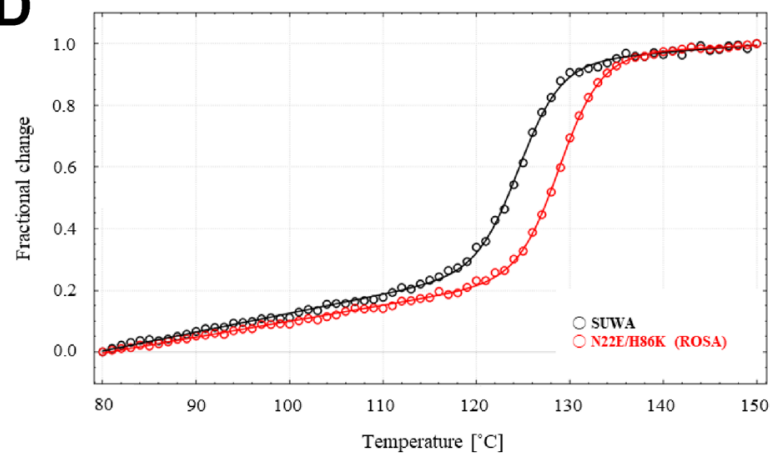

Figure 2. CD spectra and thermal denaturation curves. (A) CD spectra of WA20 and the N22A/H86K mutant of WA20 (ROWA) at 25 and $95^{\circ} \mathrm{C}$. (B) Thermal denaturation curves of WA20 and ROWA. The $T_{\mathrm{m}}$ values of the WA20 and ROWA proteins are $69.8 \pm 0.4$ and $80.4 \pm 3.5^{\circ} \mathrm{C}$, respectively. (C) CD spectra of SUWA and the N22E/ H86K mutant of SUWA (ROSA) at 30 and $150^{\circ} \mathrm{C}$. (D) Thermal denaturation curves of SUWA and ROSA. The $T_{\mathrm{m}}$ values of SUWA and ROSA are $124.7 \pm 0.1$ and $129.0 \pm 0.1^{\circ} \mathrm{C}$, respectively. In the denaturation curves, the $Y$-axis represents the normalised value of $[\theta]_{222 \mathrm{~nm}}$. The data of the denaturation curves were fitted to a two-state model with $\Delta C_{\mathrm{p}}$ fixed to zero and the graphs were created using CDpal ${ }^{33}$, version 2.18 (https://github.com/ PINT-NMR/CDpal/).

\begin{tabular}{|l|l|l|}
\hline Sample & $\boldsymbol{T}_{\mathrm{m}}\left({ }^{\circ} \mathrm{C}\right)$ & $\Delta \boldsymbol{T}_{\mathrm{m}}\left({ }^{\circ} \mathrm{C}\right)$ \\
\hline WA20 & $69.8 \pm 0.4$ & - \\
\hline N22A & $72.7 \pm 0.6$ & 2.9 \\
\hline N22E & $70.8 \pm 0.5$ & 1.0 \\
\hline N22K & $60.9 \pm 1.0$ & -8.9 \\
\hline N22L & $65.0 \pm 0.9$ & -4.8 \\
\hline H86K & $73.3 \pm 0.7$ & 3.5 \\
\hline H86S & $68.6 \pm 0.8$ & -1.2 \\
\hline H74V/S79F & $69.9 \pm 1.2$ & 0.1 \\
\hline N22A/H86K (ROWA $)$ & $80.4 \pm 3.5$ & 10.6 \\
\hline N22E/H86K & $71.7 \pm 1.1$ & 1.9 \\
\hline
\end{tabular}

Table 1. Denaturation midpoint temperatures $\left(T_{\mathrm{m}}\right)$ of the WA20 mutants.

Fig. S4). The circular dichroism (CD) spectra at $25^{\circ} \mathrm{C}$ showed that all of the mutants formed $\alpha$-helical structures, as well as the parental WA20 protein (Fig. 2A and Supplementary Fig. S5).

Thermal denaturation experiments (Table 1 and Supplementary Fig. S6) revealed that the N22A, N22E, and $\mathrm{H} 86 \mathrm{~K}$ mutants had higher midpoint temperatures than WA20 $\left(\Delta T_{\mathrm{m}}\right.$ of $2.9^{\circ} \mathrm{C}$ for $\mathrm{N} 22 \mathrm{~A}, 1.0^{\circ} \mathrm{C}$ for $\mathrm{N} 22 \mathrm{E}$, and $3.5^{\circ} \mathrm{C}$ for $\left.\mathrm{H} 86 \mathrm{~K}\right)$. In contrast, the $\mathrm{N} 22 \mathrm{~K}$ and $\mathrm{N} 22 \mathrm{~L}$ mutations considerably reduced the stability (Table 1 ).

To test combination of these stabilising mutations, we constructed two double mutants of WA20: N22A/ $\mathrm{H} 86 \mathrm{~K}$ and $\mathrm{N} 22 \mathrm{E} / \mathrm{H} 86 \mathrm{~K}$. The N22A/H86K mutant (ROWA), remarkably improved the stability $\left(\Delta T_{\mathrm{m}}=10.6^{\circ} \mathrm{C}\right)$ compared with the parental WA20 protein (Fig. 2B and Table 1). 


\begin{tabular}{|l|l|l|}
\hline Sample & $\boldsymbol{T}_{\mathrm{m}}\left({ }^{\circ} \mathbf{C}\right)$ & $\Delta \boldsymbol{T}_{\mathrm{m}}\left({ }^{\circ} \mathbf{C}\right)$ \\
\hline SUWA & $124.7 \pm 0.1$ & - \\
\hline SUWA_N22A & $128.5 \pm 0.1$ & 3.8 \\
\hline SUWA_N22E & $125.8 \pm 0.1$ & 1.1 \\
\hline SUWA_H86K & $125.6 \pm 0.3$ & 0.9 \\
\hline SUWA_N22A/H86K & $128.5 \pm 0.5$ & 3.8 \\
\hline SUWA_N22E/H86K (ROSA) & $129.0 \pm 0.1$ & 4.3 \\
\hline
\end{tabular}

Table 2. Denaturation midpoint temperatures $\left(T_{\mathrm{m}}\right)$ of the SUWA mutants.
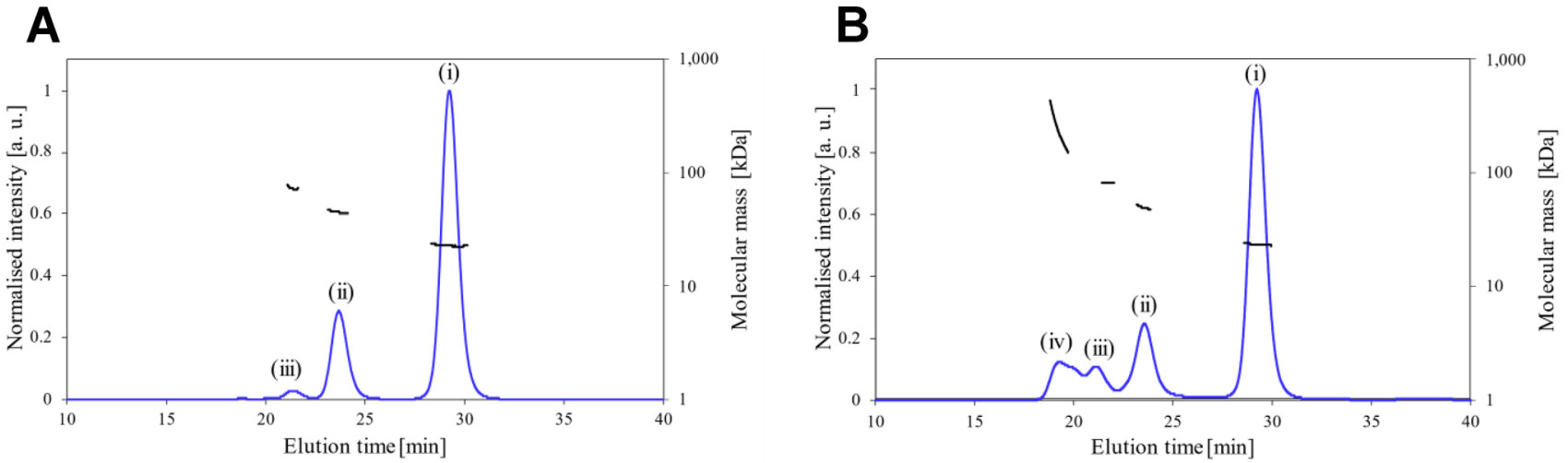

Figure 3. SEC-MALS analysis. SEC-MALS profiles of (A) ROWA and (B) ROSA. The blue lines and the black lines represent the normalised intensity of UV absorbance $\left(A_{280 \mathrm{~nm}}\right)$ and the molecular mass of the protein oligomers for each peak, respectively.

\begin{tabular}{|l|l|l|l|}
\hline Sample (peak) & $\boldsymbol{M}_{\mathbf{w}}(\mathbf{k D a})$ & Oligomeric number (mer) & Mass fraction (\%) \\
\hline ROWA (i) & 22.8 & 2 & 77 \\
\hline ROWA (ii) & 45.3 & 4 & 21 \\
\hline ROWA (iii) & 72.8 & 6 & 2 \\
\hline ROSA (i) & 23.4 & 2 & 64 \\
\hline ROSA (ii) & 49.2 & 4 & 18 \\
\hline ROSA (iii) & 81.2 & 6 & 7 \\
\hline ROSA (iv) & 238 & 19 & 11 \\
\hline
\end{tabular}

Table 3. Summary of the SEC-MALS results of ROWA and ROSA.

Development of stabilised mutants of SUWA. We also added the three stabilising mutations (N22A, $\mathrm{N} 22 \mathrm{E}$, and H86K) to SUWA, which is the hyperstable mutant (H26L/G28S/N34L/V71L/E78L) of WA20 constructed in a previous study ${ }^{20}$. Three single mutants (N22A, N22E, and H86K) and two double mutants (N22A/ $\mathrm{H} 86 \mathrm{~K}$ and N22E/H86K) of SUWA were constructed, expressed in E. coli, and purified by IMAC (Supplementary Fig. S7). For the SUWA_N22E and SUWA_N22E/H86K mutants, the yields of the purified proteins improved compared with SUWA (amount of purified protein per litre of culture of $\sim 22 \mathrm{mg}$ for SUWA_N22E, $~ 19 \mathrm{mg}$ for SUWA_N22E/H86K, and $\sim 6 \mathrm{mg}$ for SUWA). The CD spectra at $30^{\circ} \mathrm{C}$ showed that all of the mutants of SUWA formed $\alpha$-helical structures, as well as the SUWA protein (Fig. 2C and Supplementary Fig. S8). Thermal denaturation experiments revealed that all of the mutants of SUWA had higher midpoint temperatures than SUWA (Table 2, Fig. 2D, and Supplementary Fig. S6D). Compared with SUWA, the single mutations N22A, N22E, and H86K improved $T_{\mathrm{m}}$ by $3.8,1.1$, and $0.9^{\circ} \mathrm{C}$, and the double mutations $\mathrm{N} 22 \mathrm{~A} / \mathrm{H} 86 \mathrm{~K}$ and N22E/H86K improved $T_{\mathrm{m}}$ by 3.8 and $4.3^{\circ} \mathrm{C}$, respectively. We call the mutant with the highest $T_{\mathrm{m}}$ (SUWA_N22E/H86K, $T_{\mathrm{m}}=129.0^{\circ} \mathrm{C}$ ) ROSA.

Characterisation of ROWA and ROSA oligomers. To evaluate the oligomeric states of the ROWA and ROSA proteins in solution, SEC-MALS experiments were performed (Fig. 3). The molecular mass for each peak of the ROWA and ROSA proteins was determined (Table 3). The oligomeric number of each peak was calculated using the theoretical molecular mass values of monomers of ROWA $(12.5 \mathrm{kDa})$ and ROSA $(12.6 \mathrm{kDa})$. The 
A

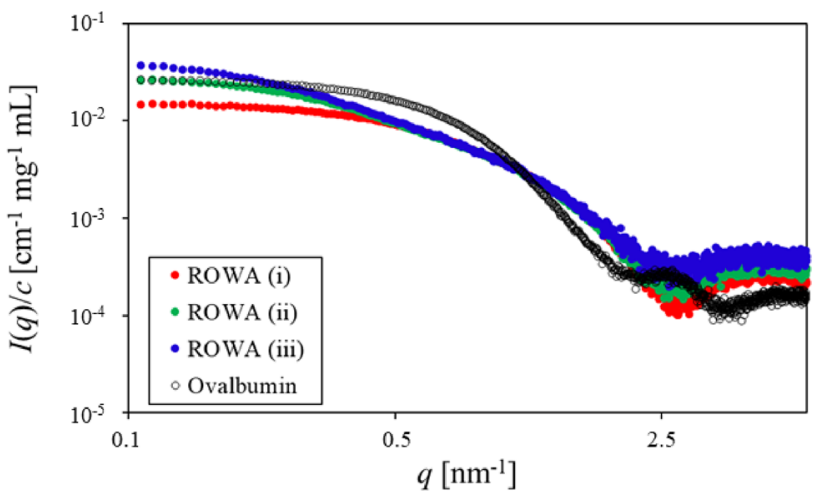

C

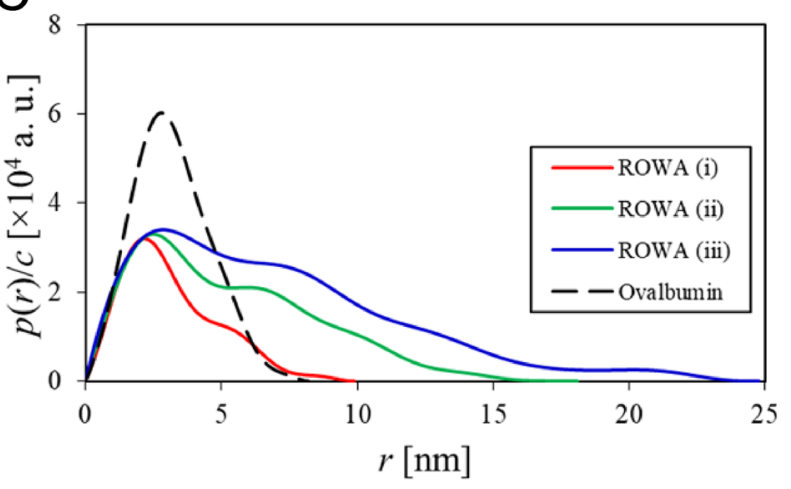

B

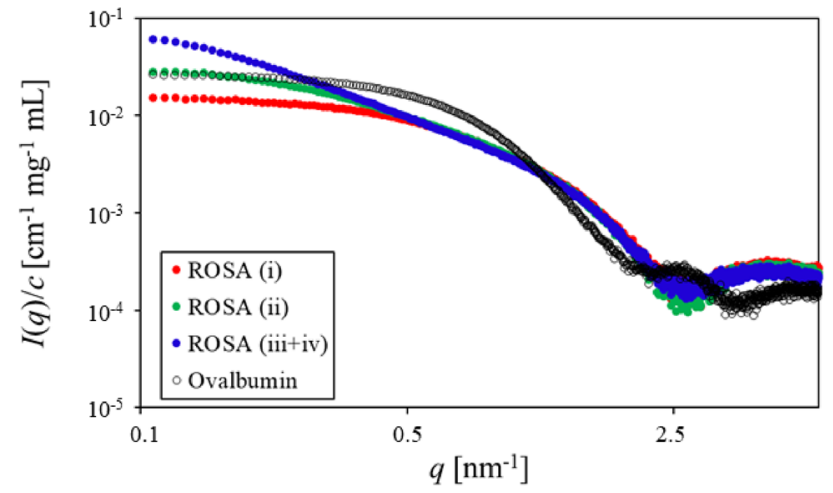

D

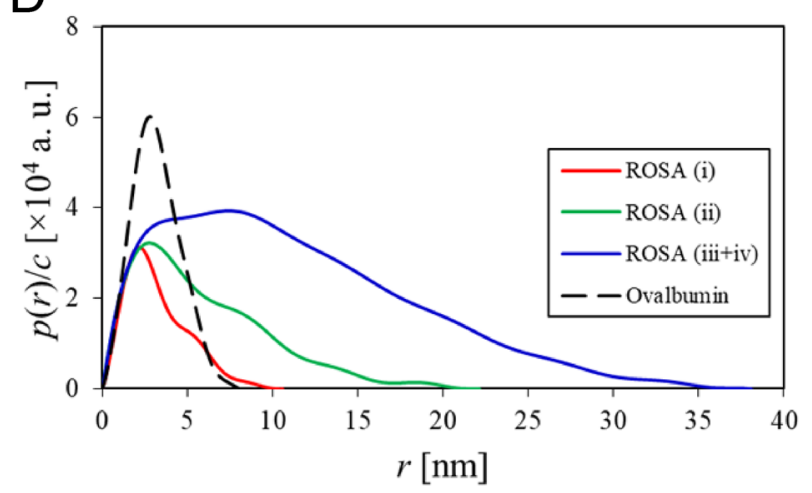

Figure 4. SAXS analysis. Concentration-normalised absolute scattering intensities of the (A) ROWA and (B) ROSA samples. Concentration-normalised pair-distance distribution functions of the (C) ROWA and (D) ROSA samples. The samples of the eluted peaks were fractionated by SEC purification as shown in Fig. 3. Ovalbumin was used as a reference standard of the molecular mass.

\begin{tabular}{|l|l|l|l|l|}
\hline Sample & $\mathbf{I}(\boldsymbol{q} \rightarrow \mathbf{0}) / \mathbf{c}\left(\mathbf{c m}^{-1} \mathbf{m g}^{-1} \mathbf{m L}\right)$ & $\boldsymbol{D}_{\max }(\mathbf{n m})$ & $\boldsymbol{R}_{\mathbf{g}}(\mathbf{n m})$ & $\boldsymbol{M}_{\mathbf{w}}(\mathbf{k D a})$ \\
\hline ROWA (i) & 0.01501 & 9.90 & 2.45 & 25.0 \\
\hline ROWA (ii) & 0.02804 & 18.1 & 4.09 & 46.8 \\
\hline ROWA (iii) & 0.04034 & 24.8 & 5.25 & 67.3 \\
\hline ROSA (i) & 0.01497 & 10.6 & 2.50 & 25.0 \\
\hline ROSA (ii) & 0.03022 & 22.1 & 4.42 & 50.4 \\
\hline Ovalbumin & 0.02657 & 8.19 & 2.45 & $44.3^{\mathrm{a}}$ \\
\hline
\end{tabular}

Table 4. Summary of the SAXS results of ROWA and ROSA. ${ }^{\mathrm{a} O v a l b u m i n}$ was used as a reference standard of the molecular mass.

ROWA protein mainly formed a dimer (peak (i), 77\%), and it also formed a tetramer (peak (ii), 21\%) and a hexamer (peak (iii), 2\%). The ROSA protein mainly formed a dimer (peak (i), 64\%), and it also formed a tetramer (peak (ii), 18\%), a hexamer (peak (iii), 7\%), and higher oligomers (peak (iv), 11\%). Although the parental WA20 and SUWA proteins only formed dimers ${ }^{16,20}$, the ROWA and ROSA proteins formed not only dimers, but also some larger oligomers (tetramers, hexamers, and higher oligomers), suggesting that the introduced mutations increase the interactions forming larger oligomers.

In addition, the SEC profiles of the isolated dimer peak fractions of ROWA and ROSA did not change after one week (Supplementary Fig. S9), suggesting that the oligomeric states are stable and do not exchange to the other oligomeric states on a timescale of a week at room temperature.

SAXS analysis. To further analyse the ROWA and ROSA oligomers, we performed SAXS experiments (Fig. 4A,B) of the samples fractionated by SEC purification. The weight-average molecular mass $\left(M_{\mathrm{w}}\right)$ values of the ROWA and ROSA samples (except for ROSA (iii + iv)) (Table 4) calculated from Guinier plots (Supplementary Fig. S10) were consistent with those from the SEC-MALS experiments (Table 3). 
A

(i)

(ii)

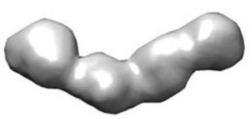

(iii)

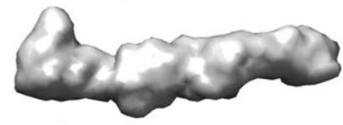

$10 \mathrm{~nm}$

B

(i)

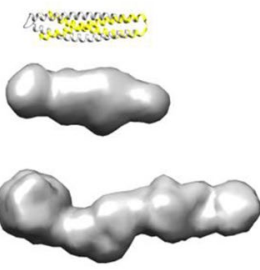

$10 \mathrm{~nm}$
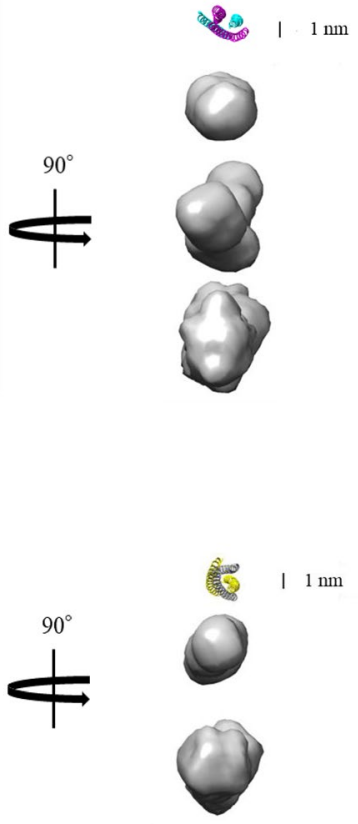

Figure 5. Dummy atom model shapes of the ROWA and ROSA oligomers. (A) Dummy atom models of the ROWA (i) dimer, (ii) tetramer, and (iii) hexamer. (B) Dummy atom models of the ROSA (i) dimer and (ii) tetramer. The models were constructed based on the SAXS data using the ab initio modelling programs $\mathrm{DAMMIF}^{40}, \mathrm{DAMAVER}^{41}$, and DAMMIN ${ }^{42}$ without symmetry constraints. Ribbon representations of the crystal structures of WA20 (PDB ID: 3VJF) ${ }^{16}$ and SUWA (PDB ID: 6KOS) ${ }^{20}$ are shown as references. These images were created using UCSF Chimera ${ }^{43}$, version 1.12 (https://www.cgl.ucsf.edu/chimera/). The SAXS data and dummy atom models have been deposited into SASBDB ${ }^{44,45}$.

To extract intuitive real-space information from the SAXS data, we obtained the pair-distance distribution functions $(p(r))$ reflected by the shapes of the ROWA and ROSA oligomers by the indirect Fourier transformation (IFT) technique (Fig. 4C,D). The shapes of $p(r)$ for all of the samples of ROWA and ROSA were characterised by an extended tail in the high- $r$ region, suggesting formation of rod-like elongated shapes similar to WA20 and SUWA. The $D_{\max }$ values of ROWA (i) (dimer), ROWA (ii) (tetramer), ROWA (iii) (hexamer), ROSA (i) (dimer), ROSA (ii) (tetramer), and ROSA (iii + iv) (hexamer and higher oligomers) indicated that the larger oligomers formed longer shapes (Fig. 4C,D and Table 4).

Low-resolution dummy atom models were reconstructed from the SAXS data (Fig. 5 and Supplementary Fig. S11). The ab initio dummy atom models of the ROWA and ROSA dimers (Fig. 5A(i),B(i)) were almost the same shapes as structures of the WA20 and SUWA dimers. Interestingly, the dummy atom models of the ROWA and ROSA tetramers (Fig. 5A(ii),B(ii)) and the ROWA hexamer (Fig. 5A(iii)) were more elongated than those of the dimers.

\section{Discussion}

Thermal denaturation is one of the main problems that limit industrial and medical use of proteins. However, experimental searches for stabilising mutations by saturation mutagenesis require enormous effort and cost because there are potentially 19 candidate amino acids at each target residue site. In this study, we attempted to discover rationally stabilising mutations of the de novo protein WA20 by MD simulation. At the four target residue sites (N22, H74, S79, and H86), we selected seven mutations of WA20 (N22A, N22E, N22K, N22L, H86K, $\mathrm{H} 86 \mathrm{~S}$, and $\mathrm{H} 74 \mathrm{~V} / \mathrm{S} 79 \mathrm{~F}$ ) based on in silico saturation mutagenesis and high-temperature MD simulations. Three mutations improving $T_{\mathrm{m}}$ of WA20 (N22A, N22E, and H86K, Table 1) were found. We then examined combinations of these mutations. While both double mutations (N22A/H86K and N22E/H86K) stabilised WA20, the contributions of these mutations to thermostabilisation may be different (Table 1). Because $\Delta T_{\mathrm{m}}$ of N22A/H86K $\left(10.6{ }^{\circ} \mathrm{C}\right)$ is greater than the sum of the $\Delta T_{\mathrm{m}}$ values of $\mathrm{N} 22 \mathrm{~A}\left(2.9^{\circ} \mathrm{C}\right)$ and $\mathrm{H} 86 \mathrm{~K}\left(3.5^{\circ} \mathrm{C}\right)$, combination of these mutations synergistically contributes to the protein stability. In contrast, $\Delta T_{\mathrm{m}}$ of $\mathrm{N} 22 \mathrm{E} / \mathrm{H} 86 \mathrm{~K}\left(1.9^{\circ} \mathrm{C}\right)$ is less than $\Delta T_{\mathrm{m}}$ of $\mathrm{H} 86 \mathrm{~K}\left(3.5^{\circ} \mathrm{C}\right)$, indicating that the combination of $\mathrm{N} 22 \mathrm{E}$ and $\mathrm{H} 86 \mathrm{~K}$ may have a negative effect for the stability of WA20.

We also added the three mutations (N22A, N22E, and H86K) to SUWA, which is the hyperstable mutant of WA20 with five mutations (H26L, G28S, N34L, V71L, and E78L) developed in a previous study ${ }^{20}$. Three single mutations (N22A, N22E, and H86K) and two double mutations (N22A/H86K and N22E/H86K) of SUWA improved $T_{\mathrm{m}}$ (Table 2). In particular, the combination of N22E and H86K mutations (ROSA) greatly improved 
the thermostability. As in the case of ROSA, $\Delta T_{\mathrm{m}}$ of $\mathrm{N} 22 \mathrm{E} / \mathrm{H} 86 \mathrm{~K}\left(4.3^{\circ} \mathrm{C}\right)$ is greater than the sum of the $\Delta T_{\mathrm{m}}$ values of $\mathrm{N} 22 \mathrm{E}\left(1.1^{\circ} \mathrm{C}\right)$ and $\mathrm{H} 86 \mathrm{~K}\left(0.9^{\circ} \mathrm{C}\right)$, suggesting that the combination of these mutations synergistically contributes to the protein stability. These results suggest that MD simulation is useful for finding stabilising mutations.

Structural models of the mutants (N22A, N22E, and H86K) of WA20 and SUWA suggest possible mechanisms for stabilisation of the structures (Supplementary Fig. S12). In the structures of the WA20 and SUWA dimers, two N22 residues are located near the R25 residues on the interface of the helices in both chains A and B (Supplementary Fig. S12A). In many natural proteins, alanine is the amino acid that has a preference to make an $\alpha$-helix ${ }^{21}$. The N22A mutation can promote formation of $\alpha$-helices and improve the structural stability. In contrast, the N22E mutation can electrostatically stabilise the protein structure by intra- and inter-chain interactions with R25 (Supplementary Fig. S12A).

Conversely, the N22K and N22L mutations destabilise the WA20 protein. Two lysine residues (N22K) and two arginine residues (R25) electrostatically repel one another (Supplementary Fig. S13). The leucine residues (N22L) may destabilise the structure probably because of exposure of large hydrophobic side chains on the interface of the helices to the solution (Supplementary Fig. S13).

In both the WA20 and SUWA structures, H86 interacts with D72 (Supplementary Fig. S12B). In neutral pH conditions, lysine has more positive charge than histidine, and thus the lysine residues (H86K) form stronger ionic bonds with the aspartic acid residues (D72), contributing to the thermostability (Supplementary Fig. S12B).

Several stabilised mutants of WA20 and SUWA, such as ROWA and ROSA developed in this study, have advantages for constructing supramolecular complexes of protein nanobuilding blocks (PN-Blocks) ${ }^{18,19}$. In particular, the ROSA protein achieved extremely high thermostability $\left(T_{\mathrm{m}}=129.0^{\circ} \mathrm{C}\right)$ and the protein expression level of ROSA in E. coli improved compared with SUWA. Therefore, ROSA is expected to improve the thermal stability and productivity of $\mathrm{PN}$-Blocks, contributing to protein engineering and synthetic biology.

\section{Methods}

Selection of the target residue sites for mutations. Candidates for the target residues for mutations of WA20 were selected based on the ASA (Table S1) in the crystal structure of WA20 (PDB ID: 3VJF) ${ }^{16} \mathrm{calcu}-$ lated by the program AREAIMOL ${ }^{22,23}$ in the CCP4 suite ${ }^{24}$. The hydrophilic residues $(\mathrm{H} 26, \mathrm{H} 74, \mathrm{E} 78, \mathrm{~S} 79$, and H86) buried inside were selected (ASA ratio to calculated GXG value $\leq 0.11$ ). In addition, target residues on the interface of $a$-helices (N22 and N34) were selected by manual inspection of the crystal structure of WA20 to potentially enhance helix-helix interactions. Because some mutations $(\mathrm{H} 26 \mathrm{~L} / \mathrm{E} 78 \mathrm{~L}$ and N34L) at the candidate residue sites had already been tested in a previous study of SUWA ${ }^{20}$, in the present study, we investigated the target residues (N22, H74, S79, and H86) that had not been previously tested.

MD simulation. The mutant structures were generated from the crystal structure of WA20 (PDB ID: $3 \mathrm{VJF})^{16}$ or SUWA (PDB ID: $\left.6 \mathrm{KOS}\right)^{20}$ using the Scwrl4 program ${ }^{25}$. The MD simulations were performed with the GROMACS 2016 molecular simulation package ${ }^{26}$. The proteins were protonated and dissolved in a dodecahedral box and placed at least $2.0 \mathrm{~nm}$ from the box edges. Periodic boundary conditions were applied in all directions. The box was filled with water molecules. Sodium and chloride ions were added to each box to neutralise the total charge. The AMBER ff14SB force field ${ }^{27}$ was used to represent the proteins and the TIP3P model ${ }^{28}$ was used for water. After energy minimisation, constant-pressure and constant-temperature (NPT) MD simulations were performed at 1 bar and $300 \mathrm{~K}$ for $0.1 \mathrm{~ns}$, and then the production runs were performed at $600 \mathrm{~K}$ for $10 \mathrm{~ns}$ with ten random seeds for each mutant in the NVT ensemble (constant temperature and volume). The Berendsen method was used to maintain the pressure during the NPT simulation ${ }^{29}$. Langevin dynamics was used to control the temperature with water viscosity set to $2 \mathrm{ps}^{-1}$. The covalent bonds of the hydrogen atoms in the proteins were constrained using the LINCS method $^{30}$, and the integration time step was $2.0 \mathrm{fs}$. During the production run, the coordinates were saved every $100 \mathrm{ps}$. The RMSD between the structures before and after the production runs was calculated by the GROMACS tool.

Construction of protein expression plasmids. The protein expression plasmids of the mutants of WA20 and SUWA were prepared by site-directed mutagenesis of the plasmid pET3-WA20 ${ }^{16}$ or pET3-SUWA ${ }^{20}$ using the transfer-PCR method ${ }^{31}$ with the oligo-DNA primers (Table S2) and KOD-Plus-Neo DNA polymerase (Toyobo, Osaka, Japan). The amino acid sequences of the WA20, ROWA, SUWA, and ROSA proteins are shown in Supplementary Fig. S14.

Protein expression and purification. The WA20, SUWA, and mutant proteins were expressed in E. coli BL21 Star (DE3) (Invitrogen, Carlsbad, CA, USA) harbouring an expression plasmid in $1 \mathrm{~L}$ of LB broth, Lennox (Nacalai Tesque, Kyoto, Japan) containing $100 \mu \mathrm{g} / \mathrm{mL}$ ampicillin sodium salt at $37^{\circ} \mathrm{C}$ for $16 \mathrm{~h}$. All the proteins were expressed in E. coli without isopropyl $\beta$-D-thiogalactopyranoside induction. The proteins were extracted from the harvested cells by freezing-thawing and sonication with a VC 505 ultrasonic processor (Sonics and Materials, Newtown, CT, USA) in $50 \mathrm{mM}$ sodium phosphate buffer ( $\mathrm{pH} 7.0$ ) containing $300 \mathrm{mM} \mathrm{NaCl}$ and $10 \%$ glycerol. The proteins were purified by immobilised metal affinity chromatography (IMAC) with TALON metal affinity resin (Takara Bio, Kusatsu, Shiga, Japan). Because many histidine residues are exposed on the surface of the WA20 and SUWA structures ${ }^{16,20}$, WA20, SUWA, and their mutants without His-tag can bind to the IMAC resin. The resin was washed with $50 \mathrm{mM}$ sodium phosphate buffer $(\mathrm{pH} 7.0)$ containing $300 \mathrm{mM} \mathrm{NaCl}$ and $10 \%$ glycerol, and the proteins were eluted with $50 \mathrm{mM}$ sodium phosphate buffer ( $\mathrm{pH} 7.0$ ) containing $300 \mathrm{mM} \mathrm{NaCl}$, $10 \%$ glycerol, and $200 \mathrm{mM}$ imidazole. The protein concentration was determined by absorbance at $280 \mathrm{~nm}$ using 
a NanoDrop Lite spectrophotometer (Thermo Fisher Scientific, Waltham, MA, USA). The molar extinction coefficient of each protein was calculated according to the amino acid sequence (Trp: 5559, Tyr: 1197, and Phe: 0.7) $)^{32}$.

CD spectroscopy. For the thermal denaturation experiments, we used a J-1500 spectropolarimeter (JASCO, Hachioji, Tokyo, Japan) specially equipped with a programmable temperature controller and a pressure-proof cell compartment that prevented the aqueous solution from boiling and evaporating at high temperatures. Thermal denaturation was monitored at the ellipticity of a typical peak of the $\alpha$-helices of proteins $\left(\theta_{222 \mathrm{~nm}}\right)$ using the cell compartment pressured by nitrogen gas $(+0.5 \mathrm{MPa})$. Each protein $(\sim 0.3 \mathrm{mg} / \mathrm{mL})$ was dissolved in $20 \mathrm{mM}$ sodium phosphate buffer ( $\mathrm{pH} 7.5$ ) containing $150 \mathrm{mM} \mathrm{NaCl}$ and $10 \%$ glycerol. A cell with $0.1 \mathrm{~cm}$ path length was used. The temperature was increased at a rate of $2.0^{\circ} \mathrm{C} / \mathrm{min}$. The thermal denaturation curves were analysed to calculate the denaturation midpoint temperatures $\left(T_{\mathrm{m}}\right)$ using $\mathrm{CDpal}^{33}$, version 2.18 . The data were fitted to a two-state model with $\Delta C_{\mathrm{p}}$ fixed to zero. The errors were estimated by the CDpal program using the robust jackknife method $^{33}$.

SEC-MALS. The SEC-MALS experiments were performed using an Alliance e2695 HPLC system (Waters, Milford, MA, USA) equipped with a Superdex 75 Increase 10/300 GL column (Cytiva, Little Chalfont, Buckinghamshire, UK), which was connected in line with a DAWN HELEOS II multi-angle static light scattering detector (Wyatt Technology, Santa Barbara, CA). The data were collected at $20^{\circ} \mathrm{C}$ with $20 \mathrm{mM}$ HEPES buffer (pH 7.5) containing $150 \mathrm{mM} \mathrm{NaCl}$ and $5 \%$ glycerol and analysed using ASTRA 6 software (Wyatt Technology) $)^{34}$. The $\mathrm{d} n /$ $\mathrm{d} c$ value of $0.185 \mathrm{~mL} / \mathrm{g}$ was generally used for the proteins with extinction coefficients of $0.542 \mathrm{~mL} \mathrm{mg}^{-1} \mathrm{~cm}^{-1}$ for ROWA and $0.539 \mathrm{~mL} \mathrm{mg}^{-1} \mathrm{~cm}^{-1}$ for ROSA calculated according to the amino acid sequences.

SAXS. For the SAXS experiments, the ROWA and ROSA samples after IMAC purification were further purified by SEC (20 mM HEPES buffer ( $\mathrm{pH} 7.5)$ containing $150 \mathrm{mM} \mathrm{NaCl}$ and $5 \%$ glycerol) with a Superdex 75 Increase 10/300 GL column (Cytiva). SAXS measurements were performed for samples $(\sim 1-4 \mathrm{mg} / \mathrm{mL})$ of ROWA, ROSA, and chicken ovalbumin (A7641; Sigma-Aldrich, St. Louis, MO, USA) dissolved in the HEPES buffer at $20^{\circ} \mathrm{C}$ using synchrotron radiation $\left(\lambda=1.3 \AA\right.$ ) at the Photon Factory BL-10C beamline ${ }^{35}$ (KEK, Tsukuba, Japan) with a PILATUS3 $2 \mathrm{M}$ detector (Dectris, Baden, Switzerland) at a sample-detector distance of $1.08 \mathrm{~m}$.

The two-dimensional scattering images were integrated into one-dimensional scattering intensities $I(q)$ as a function of the magnitude of the scattering vector $q=(4 \pi / \lambda) \sin (\theta / 2)$ using SAngler ${ }^{36}$, where $\theta$ is the total scattering angle.

The IFT technique was used to calculate the pair-distance distribution function $p(r)$ using $\mathrm{GNOM}^{37}$ in the ATSAS program suite ${ }^{38}$. The forward scattering intensity, $I(q \rightarrow 0)$, and radius of gyration, $R_{\mathrm{g}}$, were estimated by Guinier approximation ${ }^{39}$ using AUTORG in ATSAS ${ }^{38}$ with SAngler ${ }^{36}$. Assuming that the proteins have practically the same scattering length density and specific volume and that the structure factor is almost unity $(S(q)$ $\approx 1$ ) for the dilute samples, the forward scattering intensity normalised by the protein concentration $(\mathrm{mg} / \mathrm{mL})$, $I(q \rightarrow 0) / c$, is proportional to the weight average molecular mass $\left(M_{\mathrm{w}}\right)$. Ovalbumin $\left(M_{\mathrm{w}}=44.3 \mathrm{kDa}\right)$ was used as a reference standard of the molecular mass.

The low-resolution dummy atom models were constructed from the SAXS data using the ab initio shape modelling programs in the ATSAS program suite ${ }^{38}$ for small-angle scattering data analysis from biological macromolecules. The calculations of rapid ab initio shape determination were performed ten times by DAMMIF ${ }^{40}$ without a symmetry constraint, and the generated models were aligned and averaged by DAMAVER ${ }^{41}$. The averaged model was modified with the fixed core by DAMSTART and further refinement of the model was performed by DAMMIN ${ }^{42}$. The images of the dummy atom models were prepared using UCSF Chimera ${ }^{43}$, version 1.12. The SAXS data and dummy atom models of ROWA and ROSA have been deposited into Small Angle Scattering Biological Data Bank (SASBDB) ${ }^{44,45}$ (accession codes: SASDKM8 for ROWA dimer, SASDKN8 for ROWA tetramer, SASDKP8 for ROWA hexamer, SASDKQ8 for ROSA dimer, and SASDKR8 for ROSA tetramer).

Modelling of the mutant structures. The model structures of the mutants were constructed based on the crystal structure of WA20 (PDB ID: 3VJF) ${ }^{16}$ or SUWA (PDB ID: 6KOS) ${ }^{20}$ using the Scwrl4 program ${ }^{25}$. The model structures were optimised by MD simulation at $300 \mathrm{~K}$ for $1 \mathrm{~ns}$. The structure images were created using open-source PyMOL, version 2.4 (Schrödinger, New York, NY, USA).

Received: 19 December 2020; Accepted: 22 March 2021

Published online: 06 April 2021

\section{References}

1. Russell, R. J. \& Taylor, G. L. Engineering thermostability: lessons from thermophilic proteins. Curr. Opin. Biotechnol. 6, 370-374. https://doi.org/10.1016/0958-1669(95)80064-6 (1995).

2. Lehmann, M. \& Wyss, M. Engineering proteins for thermostability: the use of sequence alignments versus rational design and directed evolution. Curr. Opin. Biotechnol. 12, 371-375. https://doi.org/10.1016/s0958-1669(00)00229-9 (2001).

3. Wijma, H. J., Floor, R. J. \& Janssen, D. B. Structure- and sequence-analysis inspired engineering of proteins for enhanced thermostability. Curr. Opin. Struct. Biol. 23, 588-594. https://doi.org/10.1016/j.sbi.2013.04.008 (2013).

4. Yu, H. R. \& Huang, H. Engineering proteins for thermostability through rigidifying flexible sites. Biotechnol. Adv. 32, 308-315. https://doi.org/10.1016/j.biotechadv.2013.10.012 (2014).

5. Magliery, T. J. Protein stability: computation, sequence statistics, and new experimental methods. Curr. Opin. Struct. Biol. 33, 161-168. https://doi.org/10.1016/j.sbi.2015.09.002 (2015). 
6. Wheeler, L. C., Lim, S. A., Marqusee, S. \& Harms, M. J. The thermostability and specificity of ancient proteins. Curr. Opin. Struct. Biol. 38, 37-43. https://doi.org/10.1016/j.sbi.2016.05.015 (2016).

7. Goldenzweig, A. et al. Automated structure- and sequence-based design of proteins for high bacterial expression and stability. Mol. Cell 63, 337-346. https://doi.org/10.1016/j.molcel.2016.06.012 (2016).

8. Zimmerman, M. I. et al. Prediction of new stabilizing mutations based on mechanistic insights from Markov state models. ACS Cent. Sci. 3, 1311-1321. https://doi.org/10.1021/acscentsci.7b00465 (2017).

9. Childers, M. C. \& Daggett, V. Insights from molecular dynamics simulations for computational protein design. Mol. Syst. Des. Eng. 2, 9-33. https://doi.org/10.1039/C6ME00083E (2017).

10. Hill, R. B., Raleigh, D. P., Lombardi, A. \& Degrado, W. F. De novo design of helical bundles as models for understanding protein folding and function. Acc. Chem. Res. 33, 745-754. https://doi.org/10.1021/ar970004h (2000).

11. Smith, B. A. \& Hecht, M. H. Novel proteins: from fold to function. Curr. Opin. Chem. Biol. 15, 421-426. https://doi.org/10.1016/j. cbpa.2011.03.006 (2011).

12. Huang, P. S., Boyken, S. E. \& Baker, D. The coming of age of de novo protein design. Nature 537, 320-327. https://doi.org/10.1038/ nature19946 (2016).

13. Arai, R. Hierarchical design of artificial proteins and complexes toward synthetic structural biology. Biophys. Rev. 10, 391-410. https://doi.org/10.1007/s12551-017-0376-1 (2018).

14. Hecht, M. H., Das, A., Go, A., Bradley, L. H. \& Wei, Y. De novo proteins from designed combinatorial libraries. Protein Sci. 13, 1711-1723. https://doi.org/10.1110/ps.04690804 (2004).

15. Patel, S. C., Bradley, L. H., Jinadasa, S. P. \& Hecht, M. H. Cofactor binding and enzymatic activity in an unevolved superfamily of de novo designed 4-helix bundle proteins. Protein Sci. 18, 1388-1400. https://doi.org/10.1002/pro.147 (2009).

16. Arai, R. et al. Domain-swapped dimeric structure of a stable and functional de novo four-helix bundle protein, WA20. J. Phys. Chem. B 116, 6789-6797. https://doi.org/10.1021/jp212438h (2012).

17. Hill, R. B. \& DeGrado, W. F. Solution structure of $\alpha_{2}$ D, a nativelike de novo designed protein. J. Am. Chem. Soc. 120, 1138-1145. https://doi.org/10.1021/ja9733649 (1998).

18. Kobayashi, N. et al. Self-assembling nano-architectures created from a protein nano-building block using an intermolecularly folded dimeric de novo protein. J. Am. Chem. Soc. 137, 11285-11293. https://doi.org/10.1021/jacs.5b03593 (2015).

19. Kobayashi, N. et al. Self-assembling supramolecular nanostructures constructed from de novo extender protein nanobuilding blocks. ACS Synth. Biol. 7, 1381-1394. https://doi.org/10.1021/acssynbio.8b00007 (2018).

20. Kimura, N., Mochizuki, K., Umezawa, K., Hecht, M. H. \& Arai, R. Hyperstable de novo protein with a dimeric bisecting topology. ACS Synth. Biol. 9, 254-259. https://doi.org/10.1021/acssynbio.9b00501 (2020).

21. Pace, C. N. \& Scholtz, J. M. A helix propensity scale based on experimental studies of peptides and proteins. Biophys. J. 75, 422-427. https://doi.org/10.1016/s0006-3495(98)77529-0 (1998).

22. Lee, B. \& Richards, F. M. The interpretation of protein structures: estimation of static accessibility. J. Mol. Biol. 55, 379-400. https:// doi.org/10.1016/0022-2836(71)90324-X (1971).

23. Saff, E. B. \& Kuijlaars, A. B. J. Distributing many points on a sphere. Math. Intell. 19, 5-11. https://doi.org/10.1007/BF03024331 (1997).

24. Winn, M. D. et al. Overview of the CCP4 suite and current developments. Acta Cryst. D 67, 235-242. https://doi.org/10.1107/ S0907444910045749 (2011).

25. Krivov, G. G., Shapovalov, M. V. \& Dunbrack, R. L. Jr. Improved prediction of protein side-chain conformations with SCWRL4. Proteins 77, 778-795. https://doi.org/10.1002/prot.22488 (2009).

26. Abraham, M. J. et al. GROMACS: high performance molecular simulations through multi-level parallelism from laptops to supercomputers. SoftwareX 1-2, 19-25. https://doi.org/10.1016/j.softx.2015.06.001 (2015).

27. Maier, J. A. et al. ff14SB: Improving the accuracy of protein side chain and backbone parameters from ff99SB. J. Chem. Theory Comput. 11, 3696-3713. https://doi.org/10.1021/acs.jctc.5b00255 (2015).

28. Jorgensen, W. L., Chandrasekhar, J., Madura, J. D., Impey, R. W. \& Klein, M. L. Comparison of simple potential functions for simulating liquid water. J. Chem. Phys. 79, 926-935. https://doi.org/10.1063/1.445869 (1983).

29. Berendsen, H. J. C., Postma, J. P. M., Gunsteren, W. F. V., DiNola, A. \& Haak, J. R. Molecular dynamics with coupling to an external bath. J. Chem. Phys. 81, 3684-3690. https://doi.org/10.1063/1.448118 (1984).

30. Hess, B., Bekker, H., Berendsen, H. J. C. \& Fraaije, J. G. E. M. LINCS: A linear constraint solver for molecular simulations. J. Comput. Chem. 18, 1463-1472. https://doi.org/10.1002/(SICI)1096-987X(199709)18:12\%3c1463::AID-JCC4\%3e3.0.CO;2-H (1997).

31. Erijman, A., Dantes, A., Bernheim, R., Shifman, J. M. \& Peleg, Y. Transfer-PCR (TPCR): a highway for DNA cloning and protein engineering. J. Struct. Biol. 175, 171-177. https://doi.org/10.1016/j.jsb.2011.04.005 (2011).

32. Fasman, G. D. Practical Handbook of Biochemistry and Molecular Biology (CRC Press, 1989).

33. Niklasson, M. et al. Robust and convenient analysis of protein thermal and chemical stability. Protein Sci. 24, 2055-2062. https:// doi.org/10.1002/pro.2809 (2015).

34. Wyatt, P. J. Light-scattering and the absolute characterization of macromolecules. Anal. Chim. Acta 272, 1-40. https://doi.org/10. 1016/0003-2670(93)80373-S (1993).

35. Shimizu, N. et al. BL-10C, the small-angle x-ray scattering beamline at the photon factory. AIP Conf. Proc. 2054, 060041. https:// doi.org/10.1063/1.5084672 (2019)

36. Shimizu, N. et al. Software development for analysis of small-angle X-ray scattering data. AIP Conf. Proc. 1741, 050017. https:// doi.org/10.1063/1.4952937 (2016).

37. Svergun, D. I. Determination of the regularization parameter in indirect-transform methods using perceptual criteria. J. Appl. Crystallogr. 25, 495-503. https://doi.org/10.1107/S0021889892001663 (1992).

38. Franke, D. et al. ATSAS 2.8: a comprehensive data analysis suite for small-angle scattering from macromolecular solutions. J. Appl. Crystallogr. 50, 1212-1225. https://doi.org/10.1107/S1600576717007786 (2017).

39. Glatter, O. \& Kratky, O. Small-Angle X-Ray Scattering (Academic Press, 1982).

40. Franke, D. \& Svergun, D. I. DAMMIF, a program for rapid ab-initio shape determination in small-angle scattering. J. Appl. Crystallogr. 42, 342-346. https://doi.org/10.1107/S0021889809000338 (2009).

41. Volkov, V. V. \& Svergun, D. I. Uniqueness of ab initio shape determination in small-angle scattering. J. Appl. Crystallogr. 36, 860-864. https://doi.org/10.1107/S0021889803000268 (2003).

42. Svergun, D. I. Restoring low resolution structure of biological macromolecules from solution scattering using simulated annealing. Biophys. J. 76, 2879-2886. https://doi.org/10.1016/S0006-3495(99)77443-6 (1999).

43. Pettersen, E. F. et al. UCSF Chimera - a visualization system for exploratory research and analysis. J. Comput. Chem. 25, 1605-1612. https://doi.org/10.1002/jcc.20084 (2004).

44. Valentini, E., Kikhney, A. G., Previtali, G., Jeffries, C. M. \& Svergun, D. I. SASBDB, a repository for biological small-angle scattering data. Nucl. Acids Res. 43, D357-363. https://doi.org/10.1093/nar/gku1047 (2015).

45. Kikhney, A. G., Borges, C. R., Molodenskiy, D. S., Jeffries, C. M. \& Svergun, D. I. SASBDB: towards an automatically curated and validated repository for biological scattering data. Protein Sci. 29, 66-75. https://doi.org/10.1002/pro.3731 (2020). 


\title{
Acknowledgements
}

We thank Prof. Michael Hecht at Princeton University for the kind gift of the expression plasmid of WA20, and Dr. Naoya Kobayashi, Dr. Takahiro Kosugi, Dr. Rie Koga, and Dr. Nobuyasu Koga at the Institute for Molecular Science (IMS) for assistance with the CD experiments. The SAXS and SEC-MALS experiments were performed at Photon Factory (PF), KEK, under the approval of the PF program advisory committee (Proposal Nos. $2016 \mathrm{G} 606$ and 2018G634). We thank the beamline scientists and staff at PF, KEK. We are indebted to Research Center for Supports to Advanced Science, Shinshu University for providing facilities. We thank Dr. Tim Cooper from Edanz Group for editing a draft of this manuscript in English. This work was supported by the New Energy and Industrial Technology Development Organization (NEDO) and JSPS KAKENHI Grant Numbers JP16K05841, JP17KK0104, and JP19H02522. This work was also supported by the Joint Research Program of IMS (IMS program No. 214).

\section{Author contributions}

T.K., M.U., T.M., and R.A. designed the study. K.K., Y.S., and T.K. performed and analysed the computational calculations. S.I. performed and analysed all of the experiments. S.I., K.K., T.K., and R.A. wrote the paper. All authors discussed the results and reviewed the manuscript.

\section{Competing interests}

The authors declare no competing interests.

\section{Additional information}

Supplementary Information The online version contains supplementary material available at https://doi.org/ 10.1038/s41598-021-86952-2.

Correspondence and requests for materials should be addressed to R.A.

Reprints and permissions information is available at www.nature.com/reprints.

Publisher's note Springer Nature remains neutral with regard to jurisdictional claims in published maps and institutional affiliations.

\begin{abstract}
(c) (i) Open Access This article is licensed under a Creative Commons Attribution 4.0 International License, which permits use, sharing, adaptation, distribution and reproduction in any medium or format, as long as you give appropriate credit to the original author(s) and the source, provide a link to the Creative Commons licence, and indicate if changes were made. The images or other third party material in this article are included in the article's Creative Commons licence, unless indicated otherwise in a credit line to the material. If material is not included in the article's Creative Commons licence and your intended use is not permitted by statutory regulation or exceeds the permitted use, you will need to obtain permission directly from the copyright holder. To view a copy of this licence, visit http://creativecommons.org/licenses/by/4.0/.
\end{abstract}

(c) The Author(s) 2021 